
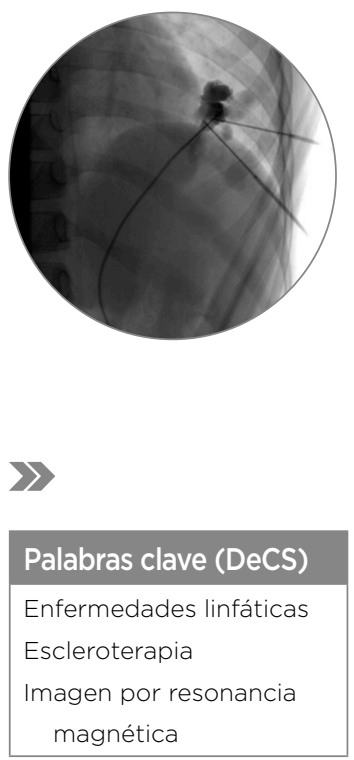

\section{Key words (MeSH)}

Lymphatic diseases

Sclerotherapy

Magnetic resonance imaging

\title{
Experiencia en el manejo de malformaciones linfáticas macroquísticas con alcohol y microquísticas con bleomicina
}

\author{
Experience In the Management of Macrocystic Lymphatic \\ Malformations with Alcohol and Microcystic Malformations with \\ Bleomycin
}

\author{
José Fernando Vallejo Díaz \\ Max Alberto Bernal Moreno ${ }^{2}$ \\ Carola Mckinster ${ }^{3}$ \\ Gerardo Montejo ${ }^{4}$
}

\section{Resumen}

Las malformaciones linfáticas son anomalías raras de flujo lento y bajo que se presentan en 1 de 6.000 a 1 en 16.000 recién nacidos vivos. Las malformaciones quísticas se clasifican según su tamaño en macroquísticas, microquísticas o mixtas. Esta clasificación tiene impacto sobre el tratamiento y el pronóstico. Las malformaciones linfáticas macroquísticas tienen mejor respuesta al tratamiento, mientras que las microquísticas son difíciles de tratar y con frecuencia recidivan. El objetivo de este trabajo es describir los resultados obtenidos en pacientes con malformaciones linfáticas macro y microquísticas intervenidos con escleroterapia utilizando alcohol y bleomicina, respectivamente. Se realizó un estudio descriptivo de pacientes con malformaciones linfáticas tratadas en el Servicio de Radiología. Se incluyeron 38 pacientes, de los cuales 24 tenían lesiones macroquísticas, 10, microquísticas y 4, mixtas. El 68 \% fueron tratados con alcohol y el 32 \% con bleomicina. El tratamiento mostró una resolución excelente en 5 pacientes, 25 tuvieron reducción del tamaño de la lesión entre 50 y 90 \%, 13 \% mostró una respuesta pobre, y solo en un $7 \%$ hubo crecimiento, a pesar del tratamiento esclerosante. De lo anterior se concluye que el tratamiento percutáneo es seguro, reduce el tamaño de las lesiones y hay pocas complicaciones informadas. Se podría utilizar como tratamiento inicial, antes de considerar una cirugía.

\section{Summary}

Lymphatic malformations are rare slow and low flow abnormalities that occur in 1 of 6,000 to 1 in 16,000 live newborns. Cystic malformations are classified according to their size as macrocystic, microcystic, or mixed. This classification has an impact on treatment and prognosis. Macrocystic lymphatic malformations have a better response to treatment, while microcystic malformations are difficult to treat and frequently recur. The objective of this study is to describe the results obtained in patients with macro and microcystic lymphatic malformations who underwent sclerotherapy using alcohol and Bleomycin, respectively. A descriptive study of patients with lymphatic malformations treated in the Radiology Service of the National Institute of Pediatrics was carried out during the period from 2014 to 2016 . Thirty-eight patients were included, 24 with macrocystic, 10 microcystic and 4 mixed lesions. $68 \%$ were treated with alcohol and $32 \%$ of the patients were treated with Bleomycin. The treatment showed excellent resolution in 5 patients, 25 patients had a lesion size reduction between 50 and $90 \%, 13 \%$ had a poor response, and only $7 \%$ had growth despite sclerosing treatment. From the above we conclude that percutaneous treatment is safe, reduces the size of the lesions and there are few reported complications. It could be used as initial treatment before considering surgery.

'Radiólogo pediatra, Clínica Centro Médico Imbanaco. Cali, Colombia.

${ }^{2}$ Radiólogo pediatra, Instituto Nacional de Pediatría. México.

${ }^{3}$ Dermatóloga pediatra, Instituto Nacional de Pediatría. México.

${ }^{4}$ Radiólogo intervencionista, Instituto Nacional de Pediatría. México.

\section{Introducción}

Las malformaciones linfáticas (ML) son anomalías raras con flujo lento y bajo. Se presentan en 1 de 6.000 a 16.000 recién nacidos vivos y se manifiestan clínicamente como neoformaciones subcutáneas del color de la piel, de consistencia blanda, renitente, sin trayectos venosos visibles. Pueden localizarse en cualquier sitio, aunque predominan en cabeza y cuello. Generalmente son asintomáticas; sin embargo, cuando afectan las vías aéreas superiores pueden obstruir la respiración. Aparecen desde el nacimiento, pero pueden manifestarse y hacerse visibles a edades más tardías cuando se asocian a infecciones de vías aéreas superiores, en el caso de 
las que asientan en cuello, o de algún traumatismo, cuando están en otras localizaciones.

Las ML se clasifican en macroquísticas, microquísticas o mixtas $(1,2)$. Esta clasificación determina el tratamiento y el pronóstico. Las malformaciones macroquísticas tienen mejor respuesta al tratamiento, principalmente tratadas con esclerosis, con la aplicación percutánea de alcohol absoluto. Las lesiones microquísticas son difíciles de tratar, pueden tener recidivas y en su manejo ha demostrado ser útil la aplicación intralesional de bleomicina (3-5).

El tratamiento percutáneo con substancias esclerosantes ha demostrado mejores resultados que los procedimientos quirúrgicos en este tipo de lesiones, ya que la resección quirúrgica provoca mayor recurrencia $(5,6)$.

Por lo tanto, es importante conocer la experiencia sobre este tipo de lesiones y su resultado con el tratamiento percutáneo.

\section{Diseño del estudio}

Se realizó un estudio retrospectivo por el periodo comprendido entre el 1 de enero de 2014 y el 30 de junio de 2016.

La población objeto fueron pacientes pediátricos con diagnóstico clínico e imagenológico de malformación linfática (ML) macroquística, microquística o mixta, atendidos en el Servicio de Radiología mediante procedimiento percutáneo y aplicación de alcohol o bleomicina.

Se incluyeron pacientes registrados en el libro de estadística de procedimientos percutáneos realizados en el Servicio de Radiología. Se seleccionaron 86 pacientes con malformaciones linfáticas a partir del informe del radiólogo intervencionista, 41 pacientes fueron excluidos cuando las imágenes de ultrasonido o resonancia diagnosticaron malformaciones venosas o los pacientes tuvieron tratamiento quirúrgico previo. Se obtuvieron datos de 45 pacientes que cumplieron los criterios de inclusión, los cuales se sometieron a comparación con la base de datos del Servicio de Dermatología; como resultado, se excluyeron 7 pacientes por tener características clínicas de malformaciones venosas o cicatrices en el lugar de la malformación. Finalmente, la muestra quedó conformada por 38 pacientes.

Los criterios de inclusión fueron: pacientes con malformaciones linfáticas quísticas de cualquier tamaño, de ambos sexos, menores de 18 años, con estudios de imagen del Instituto Nacional de Pediatría (INP), con escleroterapia en el Servicio de Radiología, con alcohol o bleomicina, y registrados en la base de datos del Servicio.

Los criterios de exclusión fueron: pacientes que hubieran sido intervenidos quirúrgicamente, tratados con esclerosantes diferentes a alcohol o bleomicina, pacientes en quienes el tratamiento esclerosante pusiera en riesgo su vida por la localización de la malformación y aquellos en quienes el tratamiento pusiera en riesgo la funcionalidad del órgano.

Los estudios debían incluir ultrasonido, resonancia magnética nuclear o ambos. Con las imágenes de ultrasonido se calculó el volumen de la lesión antes y después del procedimiento; posteriormente, se determinó el porcentaje de reducción de la lesión para evaluar su respuesta. Se hizo control ultrasonográfico entre los 60 y los 120 días posteriores al procedimiento.

Las lesiones macroquísticas se trataron con aplicación percutánea de alcohol y las lesiones microquísticas con bleomicina. Las lesiones mixtas fueron tratadas con ambos agentes.
Los procedimientos fueron realizados por dos radiólogos pediatras intervencionistas con experiencia en este tipo de tratamientos. Para la localización de las lesiones se utilizó guía ecográfica y posteriormente guía fluoroscópica; para evidenciar la ausencia de comunicación entre los espacios quísticos y las estructuras vasculares, inicialmente se instiló medio de contraste intralesional para determinar el volumen aproximado, y se colocó el $10 \%$ de lo señalado por el medio de contraste o del volumen aspirado sin sobrepasar la dosis máxima del esclerosante. El procedimiento siempre se realizó bajo anestesia general. Para la punción se utilizaron agujas de $25 \mathrm{G}$ con microset o yelcos 22 G. Un mes después del procedimiento se realizó un control con ultrasonido (US) y se aplicó una nueva dosis, si la lesión medía todavía más de $1 \mathrm{~cm}$. No se administró antibiótico profiláctico de rutina. El manejo fue ambulatorio, con salida 6 horas después del procedimiento.

En las lesiones microquísticas no se aspiró el contenido. Debido a la dificultad para canular una lesión microquística, solo se administró el medicamento en una o dos regiones de acuerdo con su extensión, con base en el volumen de la lesión medida en ecografía. Se administró un volumen correspondiente al $10 \%$ de la lesión.

Los datos fueron registrados en una base de datos de Excel y Google Drive, y analizados en el programa estadístico STATA.

La respuesta al tratamiento se midió con base en la disminución del tamaño de la lesión. Fue considerado excelente cuando hubo resolución entre el $91 \%$ y el $100 \%$; buena, cuando la resolución fue entre el $50 \%$ y el $90 \%$; pobre, con disminución inferior al $50 \%$ y mala, cuando no hubo cambios o aumentó la lesión.

\section{Análisis y medición de variables}

En este estudio se describen las variables de frecuencia de sexo, edad, tipo de lesión, localización y porcentaje de disminución de la lesión según el tipo de ML y el medicamento utilizado, con un análisis estadístico basado en frecuencias absolutas y relativas.

\section{Resultados}

\subsection{Sexo}

La distribución de las malformaciones linfáticas fue similar en ambos sexos: $54 \%$ de las macroquísticas en el sexo masculino, $46 \%$ en el sexo femenino. De las 10 malformaciones linfáticas microquísticas el $60 \%$ estaban en el sexo femenino. Las malformaciones linfáticas mixtas fueron iguales para ambos sexos.

\subsection{Edad en la que se aplicó el tratamiento}

A pesar de que las malformaciones linfáticas están presentes desde el nacimiento, en muchos casos la edad en la que se manifiesta clínicamente es variable. En este estudio, el $53 \%$ recibió tratamiento antes de cumplir el año de edad, y el $77 \%$ antes de los 5 años de edad (tabla 1). 
Tabla 1. Edad en la que se aplicó el tratamiento

\begin{tabular}{|c|c|c|}
\hline Edad (años) & Número & $\%$ \\
\hline$<1$ & 20 & 53 \\
\hline $1-4$ & 9 & 24 \\
\hline $5-9$ & 6 & 16 \\
\hline$=0>0,10$ & 3 & 7 \\
\hline Total & 38 & 100 \\
\hline
\end{tabular}

\subsection{Localización anatómica de las malformaciones linfáticas}

La distribución de frecuencia de la tabla 2, muestra que el $56 \%$ (21 casos) de las malformaciones linfáticas se localizan en cara y cuello: $9(24 \%)$ en cara, $12(32 \%)$ en cuello, $10(26 \%)$ en tórax, $3(8 \%)$ en abdomen y $4(10 \%)$ en extremidades.

Tabla 2. Localización anatómica de las malformaciones linfáticas

\begin{tabular}{|c|c|c|}
\hline Localización & Número & $\%$ \\
\hline Cara & 9 & 24 \\
\hline Cuello & 12 & 32 \\
\hline Tórax & 10 & 26 \\
\hline Abdomen & 3 & 8 \\
\hline Extremidades & 4 & 10 \\
\hline Total & 38 & 100 \\
\hline
\end{tabular}

\subsection{Tipo de malformación linfática}

Las malformaciones macroquísticas son las más frecuentes, seguidas por las microquísticas y las mixtas. En algunos casos, las malformaciones macroquísticas después de un tratamiento esclerosante pueden comportarse como mixtas. En este estudio, 24 (63\%) fueron macroquísticas, 10 (26\%), microquísticas y 4 (11\%) mixtas.

La resonancia magnética (RM) en algunos casos se utilizó como complemento inicial para evaluar la extensión de la lesión y para dar soporte al planeamiento terapéutico. Se realizó estudio con US en el $74 \%$ de los casos y RM en el $26 \%$. El ultrasonido aportó mayor información sobre las dimensiones de la lesión y su extensión, dado que la mayoría son irregulares, y se utilizó en diagnóstico, guía terapéutica y control postratamiento (figuras 1,2 y 3). De los 10 estudios de RM, 4 casos correspondieron a malformaciones macroquísticas, 3 a microquísticas y 3 a mixtas.

\subsection{Tratamiento}

Se utilizó tratamiento percutáneo con bleomicina y/o alcohol en todos los casos. El alcohol se usó en pacientes con malformaciones linfáticas macroquísticas, o cuando eran mixtas y su principal componente eran quistes grandes. La dosis administrada fue hasta máximo $1 \mathrm{~cm}^{3}$ por kilogramo de peso; en pacientes con peso menor a $10 \mathrm{~kg}$, en quienes la dosis debía ser muy baja, se administraron 1 a $2 \mathrm{~cm}^{3}$, por el riesgo de necrosis, por tratarse de un área de superficie corporal más pequeña.

La bleomicina se usó en las lesiones microquísticas o cuando el principal componente era fibroso (figura 4 ). Su presentación de $15 \mathrm{U}$ (Unidades) se diluyó en $8 \mathrm{~cm}^{3}$ de solución salina normal (SSN) y $6 \mathrm{~cm}^{3}$ de medio de contraste hidrosoluble, para obtener $1 \mathrm{U} / \mathrm{cm}^{3}$. Se administró un máximo de $1 \mathrm{~cm}^{3} / \mathrm{kg}$ por sesión sin superar $15 \mathrm{U}$ por sesión. El procedimiento se realizó bajo anestesia general y no se tomó radiografía de tórax de rutina, la cual estaba prevista si el paciente manifestaba algún síntoma respiratorio, que en nuestros pacientes no ocurrió.

La mayoría de malformaciones mixtas se trataron con aplicación de alcohol inicialmente, para manejar el componente macroquístico, y con bleomicina posteriormente, para manejar el componente microquístico. En 26 casos (68\%), el tratamiento esclerosante utilizado fue alcohol, y en 12 casos (32\%), bleomicina.

La respuesta al tratamiento fue buena en 25 pacientes $(66 \%)$ de los casos, excelente en $5(13 \%)$, pobre en $5(13 \%)$ y mala en $3(7 \%)$.

Los pacientes que tuvieron mejor respuesta al tratamiento tenían una malformación linfática macroquística localizada en el cuello, y de estos 2 tuvieron curación total.

Los 5 casos que tuvieron una respuesta menor al $50 \%$ se localizaron 3 de ellos en el tórax y 2 en la cara. Cuatro recibieron escleroterapia con alcohol absoluto y uno con bleomicina. Tres recibieron tratamiento quirúrgico posterior a la escleroterapia, por no lograr los resultados esperados.

De los casos que empeoraron, uno tenía una malformación microquística en la región axilar derecha y a pesar del manejo esclerosante aumentó su volumen (figura 5). El segundo fue una malformación linfática microquística en la lengua y el tercero localizado en el arco superciliar, también microquística. Los tres recibieron tratamiento inicial con bleomicina y por aumento del tamaño fueron sometidos a tratamiento quirúrgico.

\section{Discusión}

La clasificación de las anomalías vasculares propuesta por Mulliken and Glowacki en 1982 está basada en las características histopatológicas, separadas en dos grandes grupos. Este sistema fue adoptado por la Sociedad Internacional para el Estudio de Anomalías (ISSVA, por sus siglas en inglés), que las divide en neoplasias vasoproliferativas y malformaciones vasculares (6-9). Los términos como linfangioma, hemolinfangioma e higroma quístico se eliminaron y se reemplazaron por malformaciones linfáticas.

La clasificación de las malformaciones linfáticas se divide en comunes (quísticas), sindromáticas y linfedema primario. Las primeras, a su vez, están subdivididas según el tamaño de los quistes - macroquísticas, si los quistes son mayores de $2 \mathrm{~cm}$, microquísticas si son menores de $2 \mathrm{~cm}$, o mixtas si hay de diversos tamaños (9-11) - Estos quistes pueden estar llenos de material proteínico y eosinófilos. Otros tipos menos comunes son la anomalía linfática generalizada, la enfermedad de Gorham-Stout, la malformación linfática tipo canal y el linfedema primario (6,11-14).

Las malformaciones linfáticas se localizan principalmente en cara y cuello, seguidas por las extremidades, tronco, órganos internos, huesos y músculo. Se ha documentado que entre el $48 \%$ y el $75 \%$ de las malformaciones linfáticas se encuentran en cabeza y cuello, de las cuales la mayoría son macroquísticas (15). 
Hay un incremento en la incidencia, si están asociadas a síndrome de Turner (9,14,16-19).

Aproximadamente, el $25 \%$ de las malformaciones linfáticas ocurren en la axila. Rara vez afectan la pared intestinal o el pulmón, causando hipoproteinemia severa, pérdida de volumen, ascitis y líquido pleural $(9,14,16,20)$.

La malformación linfática puede no ser evidente al nacimiento y observarse posteriormente en la infancia o adolescencia asociada a un trauma o infección, generalmente de vías aéreas superiores; están presentes al nacimiento en el $65 \%$ de los casos y alrededor de los 2 años de edad en un 80 a $90 \%$. No hay predilección por sexo o etnia. Los síntomas más frecuentes son aumento de volumen bien delimitado, con una piel sin alteraciones, de superficie lisa, que ocasiona deformidad de la región. Las lesiones en cuello pueden producir compresión vascular o de vías aéreas, disfagia o síntomas de compresión nerviosa $(21,22)$. Característicamente, este tipo de lesiones crecen con el niño; cuando la malformación linfática está asociado a hipertrofia de partes blandas — como en el síndrome de Klipell-Trenaunay_, en la piel se puede observar dilatación de linfáticos cutáneos superficiales caracterizados por pequeñas vesículas translúcidas o violáceas milimétricas provocadas por aumento de presión de linfáticos más profundos. Las lesiones gigantes pueden presentar pérdida hídrica, hipoproteinemia o ambos. Si la lesión está asociada a una infección, cursa con crecimiento rápido y/o sangrado interno.
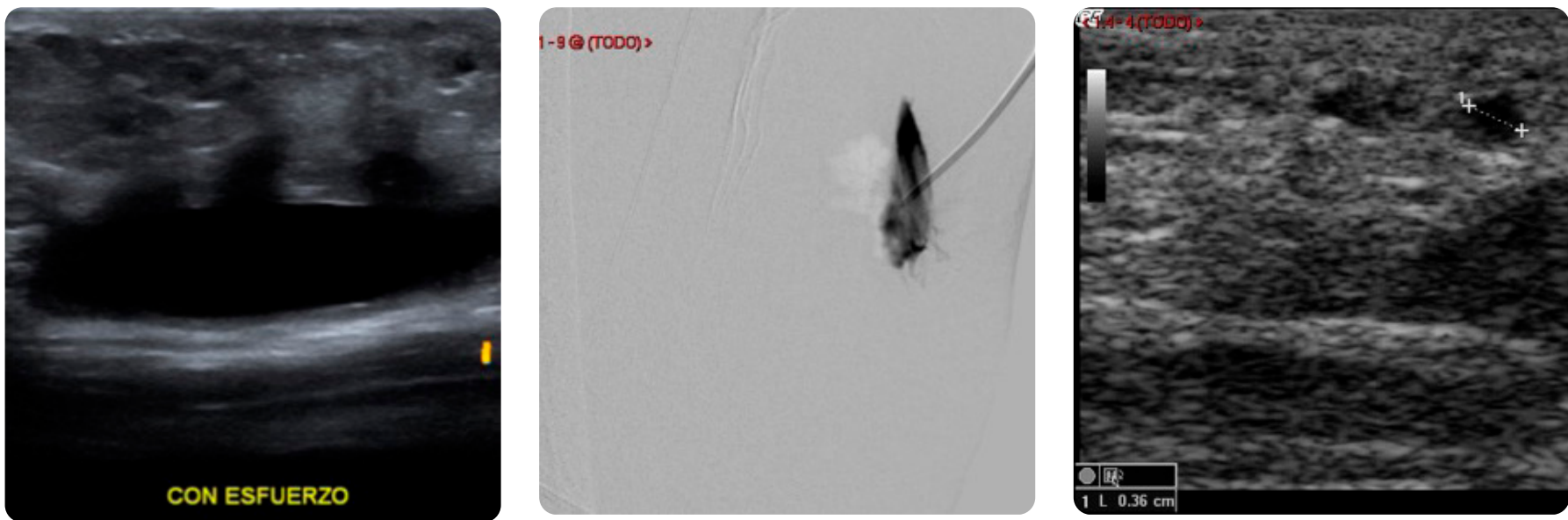

Figura 1. a) Malformación linfática macroquística en el muslo derecho: ultrasonido de alta frecuencia, una lesión anecoica de paredes bien definidas, consistencia blanda, que incrementa su tamaño con la realización de maniobras de Valsalva. b) Inyección con alcohol y medio de contraste de la malformación macroquística para escleroterapia, bajo visión fluoroscópica. c) Disminución significativa de la malformación linfática macroquística, se encontró un pequeño quiste residual.
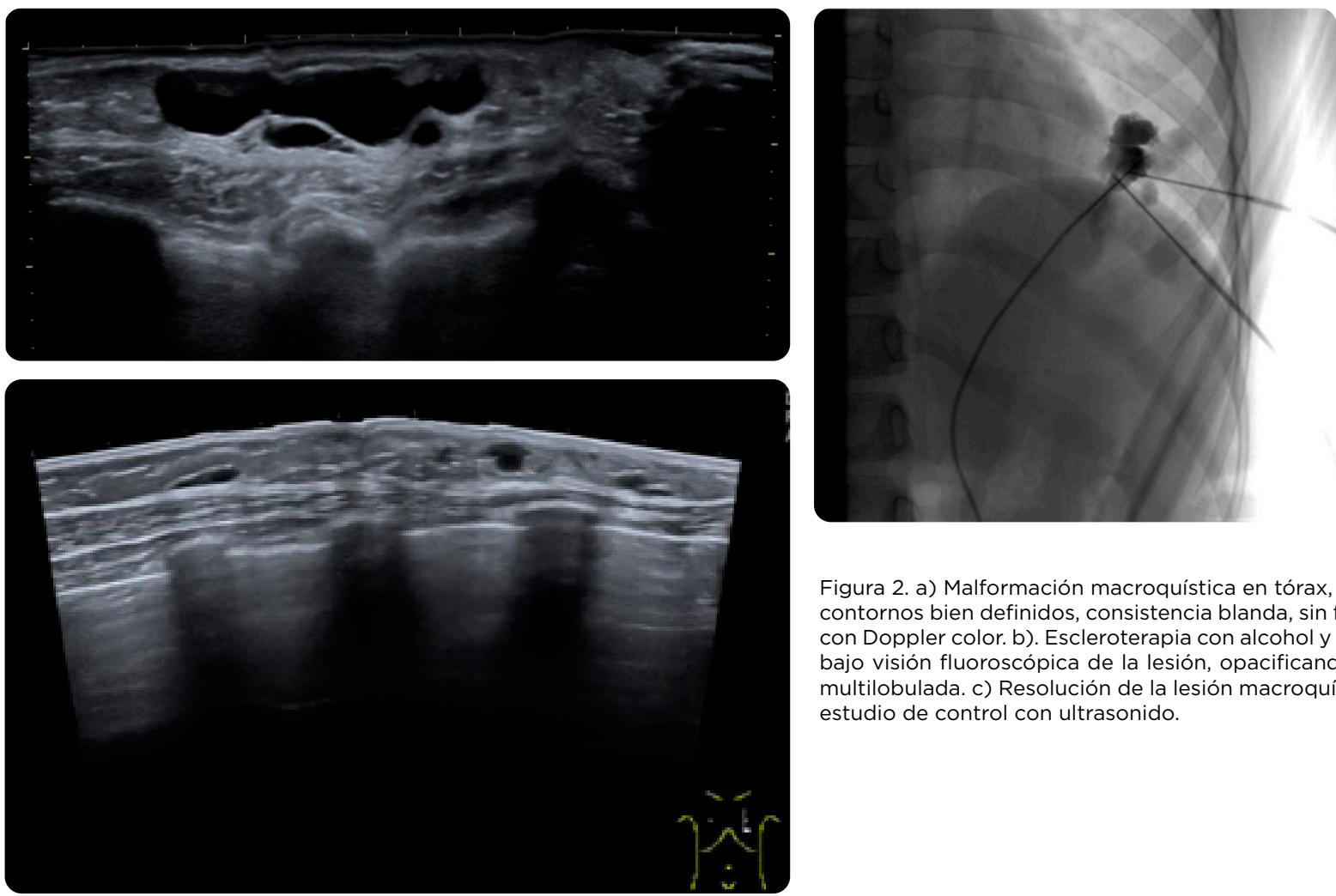

Figura 2. a) Malformación macroquística en tórax, imagen ovalada de contornos bien definidos, consistencia blanda, sin flujo a la insonación con Doppler color. b). Escleroterapia con alcohol y medio de contraste bajo visión fluoroscópica de la lesión, opacificando la lesión quística multilobulada. c) Resolución de la lesión macroquística en el tórax, en estudio de control con ultrasonido. 

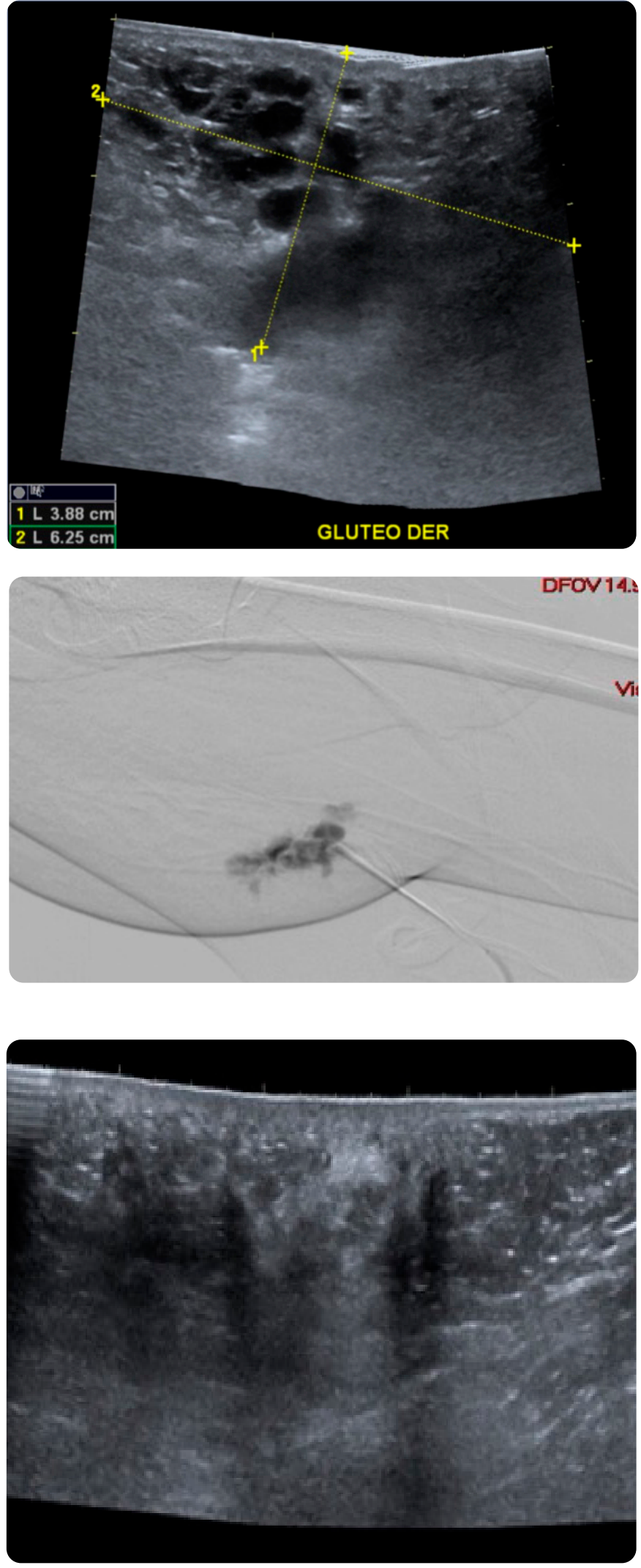

Figura 3. a) Malformación linfática mixta localizada en el glúteo derecho, ultrasonido lineal, imagen con múltiples quistes de tamaño variable, con septos finos y un componente fibroso. b) Escleroterapia con alcohol de la lesión localizada en el glúteo bajo visión fluoroscópica. c) Mejoría notoria de la lesión, con desaparición de las lesiones quísticas.
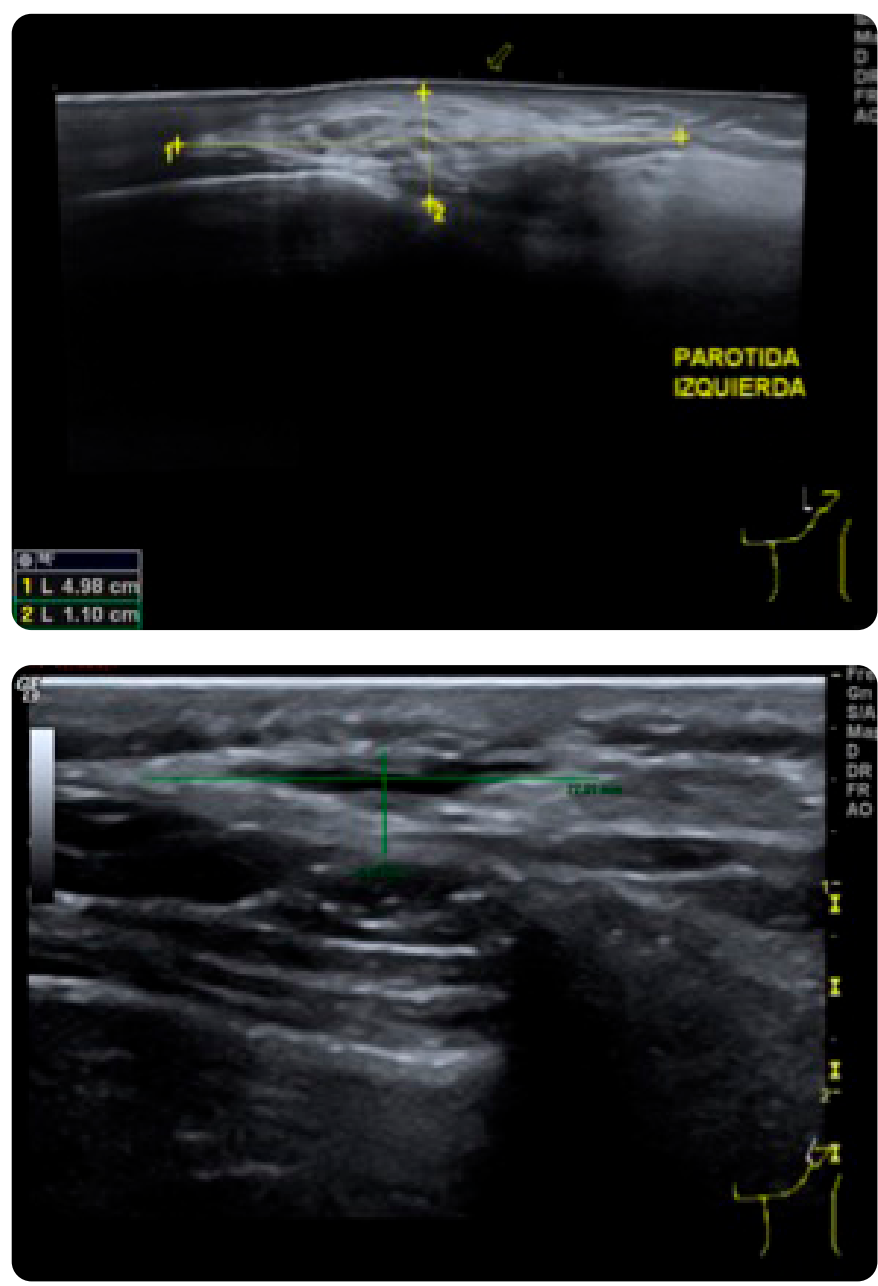

Figura 4. a). Malformación linfática microquística en la cara, ultrasonido con transductor de alta resolución: lesión ecogénica, de bordes bien definidos en parótida izquierda, con pequeños quistes sin flujo a la exploración con Doppler color, de consistencia blanda. b) Posterior escleroterapia con bleomicina: reducción en el tamaño de los microquistes, con una zona anecoica residual.

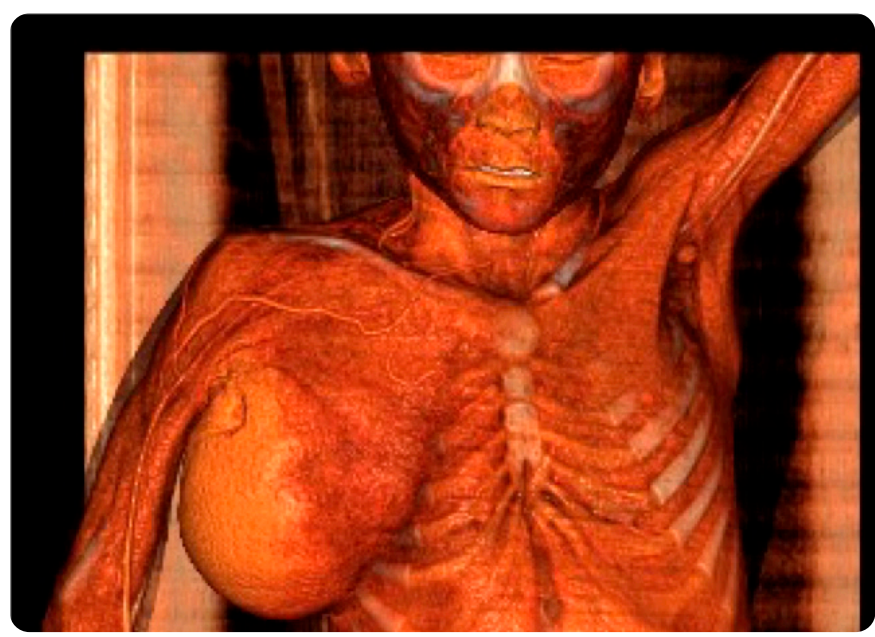

Figura 5. Reconstrucción 3D: malformación linfática microquística, sin mejoría con tratamiento esclerosante con bleomicina, tres sesiones y crecimiento de la lesión a pesar de la intervención. 
Al realizar el ultrasonido y comprimir la lesión con el transductor se observa una lesión blanda, parcialmente depresible y renitente, con tabiques internos y sin flujo en su interior; en contraste con las malformaciones arteriales, que tienen alto flujo y las malformaciones venosas, que tienen bajo flujo. La malformación macroquística puede ser única, con producción de reforzamiento acústico posterior, o tener ecos móviles en su interior en relación con detritos o hemorragia. Los tabiques son delgados, no vascularizados, puede haber escasa vascularidad periférica, excepto si está infectada, en cuyo caso hay hipervascularidad. A diferencia de la malformación venosa que puede tener grandes cambios con la respiración, cambios de posición y puede comprimirse totalmente, la malformación linfática no tiene esta característica y solo se comprime parcialmente.

En las malformaciones linfáticas microquísticas se observan pequeños quistes con numerosos tabiques. Por ser tan pequeños, los microquistes a veces son difíciles de identificar, pero producen reforzamiento acústico posterior.

La tomografía axial computarizada (TAC) y la RM delimitan mejor la extensión y muestran su relación con otras estructuras anatómicas de forma más clara que el US. La TAC muestra lesiones multiquísticas homogéneas y de baja atenuación cercana a la densidad del líquido; también ayuda a visualizar si hay extensión ósea, y es útil para la planeación quirúrgica. Cuando la lesión se infecta, se evidencia engrosamiento de las paredes de la imagen quística con aumento de la densidad interior o reforzamiento de la lesión $(15,23,24)$.

En la RM, dependiendo del contenido hemático o proteináceo, la imagen es homogénea o heterogénea con diferentes grados de intensidad en T1 según su componente. En imágenes ponderadas en T2 son marcadamente hiperintensos, comúnmente con nivel líquido dentro del quiste, principalmente en malformaciones linfáticas macroquísticas, y con componente sólido en las microquísticas. Después de la administración de medio de contraste no hay realce, o este es mínimo y localizado en anillo o periférico, o distribuido en los tabiques (25-27).

El manejo de las malformaciones linfáticas está dirigido a mantener la funcionalidad regional, disminuir los síntomas y preservar la integridad estética. El manejo debe ser realizado por un grupo interdisciplinario de especialistas que incluya un dermatólogo, un cirujano pediatra, un cirujano oncólogo, un cirujano plástico y un radiólogo intervencionista. Pueden estar integrados también especialistas de acuerdo con la topografía de la malformación; así, si se localiza en la órbita, deberá valorarla un oftalmólogo; en una extremidad con deformación de la misma, por un ortopedista, etc.

En general, el paciente acude por lesiones sintomáticas que producen dolor, sangrado, daño funcional y/o deformidad. El manejo quirúrgico no ha demostrado ser benéfico y solo se reserva para lesiones pequeñas. Esto se debe a que la cirugía puede provocar fibrosis y, en consecuencia, aumento de presión que genera dilatación en otros linfáticos. Los pacientes con riesgo de compresión de la vía aérea superior deberían ser sometidos a tratamiento en diferentes etapas y podrían requerir en ocasiones traqueostomía previa a la escleroterapia, si no fuera posible asegurar la permeabilidad de la vía aérea con intubación endotraqueal.

La aplicación del esclerosante se debe realizar bajo anestesia general, con una guía dirigida por imagen que puede ser ultrasonido, fluoroscopia o ambos; con aguja de 23 a $25 \mathrm{G}$ se punciona la lesión y se aspira determinada cantidad del contenido para que se forme un espacio vacío en el cual se inyecta la misma cantidad del agente esclerosante. Como efecto secundario, se produce inflamación de las paredes linfáticas, las cuales posteriormente se fusionarán. Es frecuente observar edema de tejidos blandos que dura aproximadamente 7 días. Los efectos colaterales indeseables o complicaciones son la necrosis cutánea superficial, así como la neuropatía cuando se daña un trayecto nervioso. Los mejores resultados se observan en las malformaciones linfáticas macroquísticas, ya que es más fácil extraer el contenido de una cámara grande que de varias muy pequeñas.

Las malformaciones linfáticas están presentes desde el nacimiento en la mayoría de los casos, pero pueden ser obvias más tardíamente. En la literatura se describe que del $80 \%$ al $90 \%$ se hacen evidentes antes de los 2 años de edad. No tiene prevalencia por sexo, lo cual se puso de manifiesto en este trabajo $(28,29)$; tampoco se ve una diferencia significativa entre el tipo de malformación linfática y el sexo. Aunque un estudio realizado en China con 65 pacientes mostró una relación de 1,7 a 1 (masculino a femenino), en el presente estudio la relación fue de 1:1 (30); por el contrario, en un estudio realizado en la Universidad de Oslo fue predominante en el sexo femenino: 37 hombres y 48 mujeres (31).

La mayoría de las lesiones son macroquísticas, en los diferentes estudios se documenta una frecuencia entre 49 y $52 \%$; en este estudio también fueron macroquísticas el $63 \%$, microquísticas $26 \%$ y mixtas $11 \%$.

En diferentes artículos se habla de una presentación en cabeza y cuello entre 48 y $75 \%$, el $56 \%$ de los pacientes aquí estudiados tenían esta localización (32-34). La mayoría son submandibulares o ubicados de forma difusa en el cuello, con extensión al mediastino. Las malformaciones en el cuello tienen buena respuesta al tratamiento. En este estudio todos los pacientes mostraron una respuesta favorable y tres de ellos tuvieron una curación total, de estos, dos eran malformaciones linfáticas macroquísticas y una mixta.

Para el diagnóstico se utilizó el US y la RM. El ultrasonido tiene la ventaja de que no es necesario sedar al paciente y se hizo previo al procedimiento, pero con la limitante de que en lesiones profundas o mayores a 10 $\mathrm{cm}$, se dificultaba obtener el campo visual para abarcar toda la imagen. $\mathrm{La}$ $\mathrm{RM}$, en cambio, brinda una adecuada definición de la lesión, así como su extensión, lo que facilitó un tratamiento quirúrgico; así mismo, el control posterior al tratamiento fue mucho más objetivo, ya que se podía observar la lesión en extenso y su reducción. Sin embargo, la RM tiene como desventajas que su alto costo de utilización aumenta el valor del estudio para el paciente, está poco disponible y requiere sedación general en pacientes menores a 5 años. En los diferentes estudios no profundizan mucho acerca de la modalidad de imágenes ni los controles posquirúrgicos (35-37).

En el trabajo realizado por Yang et al., con 65 pacientes en quienes aplicaron bleomicina en malformaciones linfáticas macroquísticas, microquísticas y mixtas, utilizaron también guía ecográfica para localizar el sitio de punción y repetían el procedimiento cada 2 a 3 semanas, independientemente del tamaño de la lesión. En los pacientes aquí estudiados, se realizó un nuevo procedimiento a las 6 semanas, solo si la cavidad era mayor a 1 cm; 32 pacientes recibieron más de una sesión de escleroterapia; 5 pacientes mejoraron con una sesión de escleroterapia, y 1 de los que recibieron 6 sesiones empeoró, a pesar del manejo.

Las lesiones que tuvieron peor respuesta se localizaban en el tórax y llama la atención que cuando empezaron su tratamiento esclerosante eran mayores a $10 \mathrm{~cm}$; en cambio, lesiones pequeñas tuvieron buena respuesta en el tórax. Sería importante determinar si en estudios futuros es reproducible este tipo de comportamiento y se pueda brindar otra clase de manejo inicial a estos pacientes, ya que no les serviría el tratamiento esclerosante.

Entre los síntomas asociados a las malformaciones vasculares linfáticas se describió parálisis facial, xeroftalmia, ptosis palpebral, dismetría de miembros pélvicos — aparentemente de mayor tamaño el miembro pélvico 
afectado por la malformación- No se especificó en qué momento apareció la lesión, por lo cual no se pudo determinar si fue secundario a la escleroterapia o al crecimiento de la malformación previo al tratamiento. Los seromas fueron la única complicación descrita asociada al procedimiento en el presente estudio.

En muchas ocasiones, y en la experiencia del grupo interdisciplinario, se encontraron recidivas de las lesiones o aparecían en el lado contralateral donde previamente había recibido tratamiento esclerosante; en el estudio no fue evidente esta característica, porque solo se hizo seguimiento durante dos años, pero en una extensión del estudio se podría determinar si en al menos diez años esta característica puede ser documentada, así como el tipo de malformación linfática y las características en cuanto a tamaño, localización y edad al inicio del tratamiento, para brindar una mejor atención a los pacientes.

\section{Conclusiones}

Las malformaciones linfáticas son entidades complejas con secuelas físicas y distorsión de la anatomía, que pueden alterar el desarrollo psicológico y afectar la función del órgano comprometido, por eso el tratamiento temprano y multidisciplinario es necesario, para brindarle al paciente las menores secuelas por su patología. El tratamiento percutáneo es efectivo y reduce las lesiones en su tamaño, es seguro y con pocas complicaciones descritas, por lo que se debería considerar como abordaje inicial, antes de la cirugía.

\section{Agradecimientos}

Rafael Glikstein. Neurorradiólogo, Universidad de Ottawa.

Katherine Vallejo Díaz. Médica y cirujana, Universidad del Valle.

\section{Referencias}

1. Garzon MC, Huang JT, Enjolras O, Frieden IJ. Vascular malformations: Part I. J Am Acad Dermatol. 2007;56(3):353-70; quiz 71-4.

2. Puig S, Casati B, Staudenherz A, Paya K. Vascular low-flow malformations in children: current concepts for classification, diagnosis and therapy. Eur J Radiol. 2005;53(1):35-45.

3. Baskin D, Tander B, Bankaoğlu M. Local bleomycin injection in the treatment of lymphangioma. Eur J Pediatr Surg. 2005;15(6):383-6.

4. Yetiser S, Karaman K. Treatment of lymphangioma of the face with intralesional bleomycin: case discussion and literature review. J Maxillofac Oral Surg. 2011;10(2):152-4.

5. Perkins JA, Manning SC, Tempero RM, Cunningham MJ, Edmonds JL, Hoffer FA, et al. Lymphatic malformations: review of current treatment. Otolaryngol Head Neck Surg. 2010;142(6):795-803

6. Acevedo JL, Shah RK, Brietzke SE. Nonsurgical therapies for lymphangiomas: a systematic review. Otolaryngol Head Neck Surg. 2008;138(4):418-24.

7. Defnet AM, Bagrodia N, Hernández SL, Gwilliam N, Kandel JJ. Pediatric lymphatic malformations: evolving understanding and therapeutic options. Pediatr Surg Int. 2016;32(5):425-33.

8. Eivazi B, Werner JA. [Extracranial vascular anomalies (hemangiomas and vascular malformations) in children and adolescents--diagnosis, clinic, and therapy]. Laryngorhinootologie. 2014;93 Suppl 1:S185-202.

9. Adams MT, Saltzman B, Perkins JA. Head and neck lymphatic malformation treatment: a systematic review. Otolaryngol Head Neck Surg. 2012;147(4):627-39.

10. Elluru RG, Balakrishnan K, Padua HM. Lymphatic malformations: diagnosis and management. Semin Pediatr Surg. 2014;23(4):178-85.

11. Behr GG, Johnson CM. Vascular anomalies: hemangiomas and beyond--part 2, Slowflow lesions. AJR Am J Roentgenol. 2013;200(2):423-36.

12. Licci S, Puma F, Sbaraglia M, Ascani S. Primary intrathymic lymphangioma. Am J Clin Pathol. 2014;142(5):683-8.

13. Olímpio HeO, Bustorff-Silva J, Oliveira Filho AG, Araujo KC. Cross-sectional study comparing different therapeutic modalities for cystic lymphangiomas in children. Clinics (Sao Paulo). 2014;69(8):505-8.

14. Balakrishnan K, Perkins J. Management of head and neck lymphatic malformations. Facial Plast Surg. 2012;28(6):596-602.
15. Wiegand S, Eivazi B, Zimmermann AP, Sesterhenn AM, Werner JA. Sclerotherapy of lymphangiomas of the head and neck. Head Neck. 2011;33(11):1649-55.

16. Aydin S, Demir MG, Selek A. A giant lymphangioma on the neck. J Craniofac Surg. 2015;26(4):e323-5.

17. Barnacle AM, Theodorou M, Maling SJ, Abou-Rayyah Y. Sclerotherapy treatment of orbital lymphatic malformations: a large single-centre experience. Br J Ophthalmol. 2016;100(2):204-8.

18. Ardıçlı B, Karnak İ, Çiftçi A, Tanyel FC, Şenocak ME. Sclerotherapy with bleomycin versus surgical excision for extracervical cystic lymphatic malformations in children. Surg Today. 2016;46(1):97-101.

19. Garzon MC, Huang JT, Enjolras O, Frieden IJ. Vascular malformations. Part II: associated syndromes. J Am Acad Dermatol. 2007;56(4):541-64.

20. Balakrishnan K, Bauman N, Chun RH, Darrow DH, Grimmer JF, Perkins JA, et al Standardized outcome and reporting measures in pediatric head and neck lymphatic malformations. Otolaryngol Head Neck Surg. 2015;152(5):948-53.

21. Rozman Z, Thambidorai RR, Zaleha AM, Zakaria Z, Zulfiqar MA. Lymphangioma: Is intralesional bleomycin sclerotherapy effective? Biomed Imaging Interv J. 2011;7(3):e18.

22. Cho BC, Kim JB, Lee JW, Choi KY, Yang JD, Lee SJ, et al. Cervicofacial lymphatic malformations: A retrospective review of 40 cases. Arch Plast Surg. 2016;43(1):10-8

23. Tanigawa N, Shimomatsuya $\mathrm{T}$, Takahashi K, Inomata $\mathrm{Y}$, Tanaka $\mathrm{K}$, Satomura K, et al. Treatment of cystic hygroma and lymphangioma with the use of bleomycin fat emulsion. Cancer. 1987;60(4):741-9.

24. Dubois J, Garel L, Abela A, Laberge L, Yazbeck S. Lymphangiomas in children: percutaneous sclerotherapy with an alcoholic solution of zein. Radiology. 1997;204(3):651-4

25. Ramashankar, Prabhakar C, Shah NK, Giraddi G. Lymphatic malformations: A dilemma in diagnosis and management. Contemp Clin Dent. 2014;5(1):119-22.

26. Connell F, Homfray T, Thilaganathan B, Bhide A, Jeffrey I, Hutt R, et al. Congenital vascular malformations: a series of five prenatally diagnosed cases. Am J Med Genet A. 2008;146A(20):2673-80.

27. Lev S, Lev MH. Imaging of cystic lesions. Radiol Clin North Am. 2000;38(5):1013-27.

28. Rebuffini E, Zuccarino L, Grecchi E, Carinci F, Merulla VE. Picibanil (OK-432) in the treatment of head and neck lymphangiomas in children. Dent Res J (Isfahan). 2012;9(Suppl 2):S192-6.

29. De Leacy R, Bageac DV, Manna S, Gershon BS, Kirke D, Shigematsu T, et al. A radiologic grading system for assessing the radiographic outcome of treatment in lymphatic and lymphatic-venous malformations of the head and neck. AJNR Am J Neuroradiol. 2021;42(10):1859-64.

30. Yang Y, Sun M, Ma Q, Cheng X, Ao J, Tian L, et al. Bleomycin A5 sclerotherapy for cervicofacial lymphatic malformations. J Vasc Surg. 2011;53(1):150-5.

31. Eliasson JJ, Weiss I, Høgevold HE, Oliver N, Andersen R, Try K, et al. An 8-year population description from a national treatment centre on lymphatic malformations. J Plast Surg Hand Surg. 2016:1-6.

32. Mayouego Kouam J, Epée E, Azria S, Enyama D, Omgbwa Eballe A, Ebana Mvogo $\mathrm{C}$, et al. [Epidemiological, clinical and therapeutic features of pediatric ocular injuries in an eye emergency unit in Île-de-France]. J Fr Ophtalmol. 2015;38(8):743-51.

33. Okazaki T, Iwatani S, Yanai T, Kobayashi H, Kato Y, Marusasa T, et al. Treatmen of lymphangioma in children: our experience of 128 cases. J Pediatr Surg. 2007;42(2):386-9.

34. Khunger N. Lymphatic malformations: current status. J Cutan Aesthet Surg 2010;3(3):137-8

35. Kertész Z, Bălă G, Bancu S, Gozar H, Virgil G, Horváth E, et al. [Bleomycin therapy for lymphangioma]. Chirurgia (Bucur). 2011;106(1):103-7.

36. Gorincour G, Paris M, Aschero A, Desvignes C, Bourlière B, Petit P. [Percutaneous treatment of cystic lymphangiomas]. Ann Chir Plast Esthet. 2006;51(4-5):423-8.

37. Mahady K, Thust S, Berkeley R, Stuart S, Barnacle A, Robertson F, et al. Vascular anomalies of the head and neck in children. Quant Imaging Med Surg. 2015;5(6):886-97.

\section{Correspondencia}

José Fernando Vallejo Díaz

Clínica Imbanaco

Carrera 38 Bis \# 5B2-04

Cali, Colombia

jose.vallejo@imbanaco.com.co

Recibido para evaluación: 3 de mayo de 2020

Aceptado para publicación: 10 de enero de 2021 